\title{
SARS-CoV-2 screening in pediatric cancer patients before hospitalization for chemotherapy infusion
}

\author{
M. Victoria Cabarcos ${ }^{a} \mathbb{D}$, Myriam Onoratelli ${ }^{a} \mathbb{D}$, Mariano Lorenzo $^{a}(\mathbb{D})$, Sabrina Guzmán ${ }^{a} \mathbb{D}$, Mailen Ríos $^{a}(\mathbb{D}$, \\ Marina Spilottia ${ }^{(\mathbb{D})}$, Sandra Gómez ${ }^{a}(\mathbb{D})$, Lidia Fraquellia ${ }^{(\mathbb{D})}$
}

\begin{abstract}
An observational, prospective study was carried out at Hospital Garrahan. Its objectives were to establish therateof asymptomatic severe acute respiratory syndrome coronavirus 2 (SARSCoV-2) among children with cancer and/or their caregivers during hospitalization for chemotherapy, and describe the impact of ongoing treatment among those positive for SARSCoV-2 or with symptoms compatible with infection during hospitalization.

Patients with onco-hematological disease and their caregivers were included. A reverse transcription polymerase chain reaction for SARS-CoV-2 was done.

A total of 733 hospitalizations were analyzed. The SARS-CoV-2 positivity rate was $2.2 \%$ (95\% confidence interval: 1.35-3.52). All patients with a detectable test result completed chemotherapy. Also, $7.7 \%$ of patients developed symptoms compatible with a suspected case although they had an undetectable test result, and $77 \%$ of them were able to continue treatment.

Key words: COVID-19, mass screening, neoplasms, chemotherapy.
\end{abstract}

http: / / dx.doi.org/10.5546/ aap.2022.eng.118

To cite: Cabarcos V, Onoratelli M, Lorenzo M, Guzmán S, et al. SARSCoV-2 screening in pediatric cancer patients before hospitalization for chemotherapy infusion. Arch Argent Pediatr 2022;120(2):118-121.

a. Hospital de Pediatría S.A.M.I.C. Dr. Juan P. Garrahan, Autonomous City of Buenos Aires, Argentina.

E-mail address:

M. Victoria Cabarcos: mvcabarcos@gmail.com

Funding: None.

Conflict of interest: None.

Received: 6-30-2021

Accepted: 9-6-2021

\section{INTRODUCTION}

The pandemic caused by severe acute respiratory syndrome coronavirus 2 (SARSCoV-2) has had an unprecedented impact on society and its health care systems. Cancer management has been deeply affected as a result of the barriers observed in the health care process.

According to different articles, children receiving cancer treatment usually develop asymptomatic, mild or moderate SARS-CoV-2 infection and seem to have a lower risk for severe infection compared to the general pediatric population..$^{1-5}$

According to the National Cancer Institute's Argentine Hospital Oncopediatric Registry, 417 confirmed cases were reported in the period between 4-23-2020 and 4-24-2021 among patients with onco-hematological disease. Of them, 45\% were asymptomatic. ${ }^{6}$

Available evidence highlights the need to prioritize an overall pediatric cancer care and ongoing cancer treatment in a safe or effective manner. Hospitals worldwide have implemented new policies to provide an adequate care to this population. ${ }^{1}$

Several guidelines recommend screening patients receiving chemotherapy for the early detection and isolation of asymptomatic patients..$^{7-10}$ To date, there is little information about the continuation or modification of chemotherapy in asymptomatic children with a positive test. ${ }^{11}$

One of the strategies implemented at Hospital Garrahan was introducing a screening program for asymptomatic coronavirus disease 2019 (COVID-19) prior to hospitalization for chemotherapy courses or parenteral hematological treatment. The objectives of this study were to know the rate of asymptomatic SARS-CoV-2 among children with cancer and/ or their caregivers during hospitalization for chemotherapy and describe the impact on ongoing treatment among those with detectable levels in the test or symptoms compatible with SARS-CoV-2 during hospitalization. 


\section{POPULATION AND METHODS}

This was an observational and prospective study conducted between 7-20-2020 and 4-302021.

Patients with onco-hematological disease admitted for scheduled chemotherapy or parenteral hematological treatment from an outpatient clinic and their caregivers were included.

A reverse transcription polymerase chain reaction (RT-PCR), which is a microbiological detection test, was done for SARS-CoV-2 in patients and their caregivers.

Patients hospitalized for treatment with an undetectable test result in the previous 72 hours and dyads with epidemiological discharge after a SARS-CoV-2 infection in the 3 months after diagnosis were excluded.

The underlying onco-hematological disease was classified based on the third revision of the International Classification of Diseases for Oncology by the World Health Organization and the International Classification of Childhood Cancer. Cases were grouped as follows: 1) leukemias and lymphomas, 2) solid tumors, 3) central nervous system (CNS) tumors, and 4) hematological disease.

The following variables were recorded: age, sex, diagnostic group, diagnosis, SARS-CoV-2 test result (patient and caregiver), chemotherapy interruption or modification due to the presence of clinical symptoms included in suspected case criteria with detectable and undetectable SARS$\mathrm{CoV}-2$ test results, and chemotherapy interruption or modification in asymptomatic patients with detectable levels.
Recorded clinical symptoms were those specified in the suspected case criteria published by the National Ministry of Health. ${ }^{12}$

The study was approved by the Ethics Committee and Research Review Committee of the hospital.

\section{Statistical analysis}

For the descriptive analysis of continuous variables, mean and median were used as summary statistics, based on data distribution. Categorical outcome measures were established as absolute and percent values. The statistical analysis was performed with the Epi Info® statistical software, version 7.2.4.

\section{RESULTS}

A total of 865 hospitalizations of patients for scheduled treatment and the same number of caregivers were recorded. The inclusion criteria were met by 733 hospitalizations in 241 patients who received 1 to 15 chemotherapy courses (median: 3).

Of these 241 patients, 128 were males (53\%) and their median age was 91 months (range: 1-210). Results are shown in Table 1.

Out of 733 tests done in hospitalized patients, 16 had a positive result for SARS-CoV-2. The positivity rate was $2.2 \%$ (95\% confidence interval [CI]: 1.35-3.52). All confirmed cases remained asymptomatic throughout the hospitalization and completed their chemotherapy schedule.

The positivity rate in caregivers was 2.2\% (95\% CI: 1.35-3.52). A simultaneous positivity for SARSCoV-2 was observed in 8 patient-caregiver dyads.

TABLE 1. Results of SARS-CoV-2 screening test in patients assessed in each hospitalization, characteristics of underlying disease, and results of tests in caregivers

\begin{tabular}{|c|c|c|c|c|c|c|c|c|}
\hline \multirow[t]{2}{*}{ Variable } & \multicolumn{2}{|c|}{ Total } & \multicolumn{2}{|c|}{$\begin{array}{l}\text { Detectable } \\
\text { SARS-CoV-2 }\end{array}$} & \multicolumn{2}{|c|}{$\begin{array}{l}\text { Undetectable } \\
\text { SARS-CoV-2 }\end{array}$} & \multicolumn{2}{|c|}{$\begin{array}{l}\text { Test not } \\
\text { performed }^{*}\end{array}$} \\
\hline & $\mathrm{N}$ & $\%$ & $\mathrm{~N}$ & $\%$ & $\mathrm{~N}$ & $\%$ & $\mathrm{~N}$ & $\%$ \\
\hline $\begin{array}{l}\text { Hospitalizations } \\
\text { Type of disease }\end{array}$ & 733 & & 16 & & 702 & & 15 & \\
\hline Leukemias and lymphomas & 305 & 41.6 & 7 & 43.8 & 293 & 41.7 & 5 & 33.3 \\
\hline Solid tumors & 292 & 39.8 & 9 & 56.3 & 275 & 39.2 & 8 & 53.3 \\
\hline CNS tumors & 115 & 15.7 & 0 & 0.0 & 113 & 16.1 & 2 & 13.3 \\
\hline Hematological disease & 21 & 2.9 & 0 & 0.0 & 21 & 3.0 & 0 & 0.0 \\
\hline Caregivers & 733 & & 16 & & 696 & & & 21 \\
\hline
\end{tabular}

*Test not performed: when 1 of the 2 dyad members did not meet the testing criteria. CNS: central nervous system. 
Clinical symptoms compatible with suspected case were observed in $7.8 \%$ of patients. The most common symptoms are described in Table 2.

Chemotherapy continued as scheduled in $77 \%$ of hospitalized patients with compatible symptoms. All of them had an undetectable test result for SARS-CoV-2 and remained in the prolonged chemotherapy administration unit during symptom progression.

Scheduled treatment was interrupted in 23\% of patients with symptoms compatible with SARS-CoV-2 infection.

\section{DISCUSSION}

An adequate care in pediatric oncology depends on a timely assessment and diagnosis, the referral to tertiary care facilities, the interaction among multidisciplinary teams, a coordinated multimodal management, and access to health care centers. All these factors have been affected by the pandemic.

Multiple strategies ${ }^{2}$ have been implemented worldwide to minimize the risk for SARS-CoV-2 infection in children with cancer without reducing treatment effectiveness.

Asymptomatic patients are a potential source of infection. Several articles ${ }^{7-10}$ agree that RT-PCR screening for SARS-CoV-2 allows for an early and effective detection in order to reduce viral dissemination.

Data about the prevalence of SARS-CoV-2 in pediatric cancer patients are limited and there is not enough evidence about the continuation or modification of scheduled chemotherapy in asymptomatic children with a positive test.

In our study, we observed a low rate of asymptomatic SARS-CoV-2 in children with cancer and their caregivers.

Boulad et al., ${ }^{4}$ analyzed the results of positive SARS-CoV-2 results among 3 cohorts. They described a cohort of pediatric cancer patients with asymptomatic SARS-CoV-2 who had a
RT-PCR test prior to receiving chemotherapy or sedation and the positivity rate was $2.5 \%$. This is consistent with the results of our study. They also described other cohort that included patient caregivers. In this case, the positivity rate was $14.7 \%$. This rate is much higher than that observed in our study.

We found a simultaneous positivity for SARSCoV-2 among 8 dyads. Seven patients had a negative test in spite of close contact with their positive caregivers. Unacknowledged SARS$\mathrm{CoV}-2$ infection in asymptomatic caregivers is an important consideration for infection control.

In Italy, during the peak of the pandemic, several pediatric onco-hematology centers adopted a SARS-CoV-2 screening protocol before chemotherapy initiation.

Cesaro S. et al., ${ }^{13}$ found that the prevalence of a positive test among pediatric patients who attended the hospital for chemotherapy was approximately $3 \%$, a rate that is mildly higher than that reported in our study. They also described that the strategy adopted for these patients was delaying chemotherapy until they had 2 consecutive negative tests. Such precaution mentioned by Cesaro et al., ${ }^{13}$ is consistent with the little evidence about the impact of SARS-CoV-2 on the morbidity and mortality of children with cancer at the time of publication.

Both Bisogno et al., ${ }^{14}$ and Ferrari et al.., ${ }^{15}$ suggest that cancer treatments may not need to be modified or delayed in the case of pediatric patients.

In our population, unlike what has been described by Cesaro et al., ${ }^{13}$ and consistent with what has been reported by Bisogno et al., ${ }^{14}$ and Ferrari et al., ${ }^{15}$ patients with confirmed SARSCoV-2 completed their chemotherapy without developing clinical complications. They all remained asymptomatic during hospitalization and were transferred to a specific ward assigned to the hospitalization of positive cases. Patients

TABLE 2. Symptoms compatible with SARS-CoV-2 infection among patients hospitalized for chemotherapy

\begin{tabular}{lcc}
\hline Compatible symptoms & N & $\%$ \\
\hline Isolated fever $\left(\geq 37.5^{\circ} \mathrm{C}\right)$ & 30 & 52.6 \\
Fever with hemodynamic involvement & 6 & 10.5 \\
Fever and diarrhea & 2 & 3.5 \\
Diarrhea & 19 & 33.3 \\
Total & 57 & 100.0 \\
\hline
\end{tabular}


with clinical symptoms compatible with a suspected case had an undetectable test result, and the interruption of their treatment was related to clinical criteria not associated with SARS-CoV-2.

A limitation of this study is the small number of positive asymptomatic patients, which prevents us from making a definite recommendation in relation to cancer treatment continuation. Further prospective and multicenter studies are required to better know the effects of COVID-19 on the pediatric population with onco-hematological disease.

\section{CONCLUSION}

This study shows a low prevalence of asymptomatic COVID-19 patients in our population. The data described here suggest that asymptomatic patients may continue with their treatment as scheduled.

\section{REFERENCES}

1. Millen GC, Arnold R, Cazier JB, Curley H, et al. Severity of COVID-19 in children with cancer: Report from the United Kingdom Paediatric Coronavirus Cancer Monitoring Project. Br J Cancer. 2021; 124(4):754-9.

2. Sullivan M, Bouffet E, Rodriguez-Galindo C, Fineman S, et al. The COVID-19 pandemic: A rapid global response for children with cancer from SIOP, COG, SIOP-E, SIOPPODC, IPSO, PROS, CCI, and St Jude Global. Pediatr Blood Cancer. 2020; 67(7):e28409.

3. de Rojas T, Pérez-Martínez A, Cela E, Baragaño M, et al. COVID-19infection in children and adolescents with cancer in Madrid. Pediatr Blood Cancer. 2020; 67(7):e28397.

4. Boulad F, Kamboj M, Bouvier N, Mauguen A, Kung A. COVID-19 in Children With Cancer in New York City. JAMA Oncol. 2020; 6(9):1459-60.

5. Dorantes-Acosta E, Ávila-Montiel D, Klünder M, JuárezVillegas L, Márquez-González H. Survival in pediatric patients with cancer during the COVID-19 pandemic: scoping systematic review. Bol Med Hosp Infant Mex. 2020; 77(5):234-41.

6. Moreno F, Chaplin MA. Registro oncopediátrico hospitalario argentino. $7^{\mathrm{a}}$ ed. Ciudad Autónoma de Buenos Aires: Instituto Nacional del Cáncer, 2021.

7. Rouger-Gaudichon J, Bertrand Y, Boissel N, Brethon B, et al. COVID-19 and acute lymphoblasticleukemias of children and adolescents: Updated recommendations (Version 2) of the Leukemia Committee of the French Society for the fight against Cancers and leukemias in children and adolescents (SFCE). Bull Cancer. 2021; 108(5):490-500.

8. Hanson KE, Caliendo AM, Arias CA, Englund J, et al. Infectious Diseases Society of America Guidelines on the Diagnosis of COVID-19. Clin Infect Dis. 2020; ciaa760.

9. Roques T, Board R. Guidance on SARS-CoV-2 antigen testing for asymptomatic healthcare workers (HCW) and patients in non-surgical oncology in the UK. 2020. [Accessed on: September $6^{\text {th }}$, 2021]. Available at: https: / / www.rcr.ac.uk/sites / default / files / guidance-covid19testing-asymptomatic-hcw-patients-oncology.pdf

10. Lee LYW, Hill T, Topping O, Tilby M, et al. Utility of COVID-19 Screening in Cancer Patients. Cancer Cell. 2020; 38(3):306-7.

11. Al-Shamsi HO, Coomes EA, Alrawi S. Screening for COVID-19 in Asymptomatic Patients With Cancer in a Hospital in the United Arab Emirates. JAMA Oncol. 2020; 6(10):1627-8.

12. Argentina. Ministerio de Salud. Coronavirus COVID-19: Definición de caso. [Accessed on: September $7^{\text {th }}, 2021$ ]. Available at: https://www.argentina.gob.ar/salud/ coronavirus/definicion-de-caso

13. Cesaro S, Compagno F, Zama D, Meneguello L, et al. Screening for SARS-CoV-2 infection in pediatric oncology patients during the epidemic peak in Italy. Pediatr Blood Cancer. 2020; 67(8):e28466.

14. Bisogno G, Provenzi M, Zama D, Tondo A, et al. Clinical Characteristics and Outcome of Severe Acute Respiratory Syndrome Coronavirus 2 Infection in Italian Pediatric Oncology Patients: A Study From the Infectious Diseases Working Group of the Associazione Italiana di Oncologia e Ematologia Pediatrica. J Pediatr Infect Dis Soc. 2020; 9(5):5304.

15. Ferrari A, Zecca M, Rizzari C, Porta F, et al. Children with cancer in the time of COVID-19: An 8-week report from the six pediatric onco-hematology centers in Lombardia, Italy. Pediatr Blood Cancer. 2020; 67(8):e28410. 